Applied Remote Sensing

Errata: Mapping soil water content under sparse vegetation and changeable sky conditions: comparison of two thermal inertia approaches

\author{
Antonino Maltese \\ Fulvio Capodici \\ Giuseppe Ciraolo \\ Goffredo La Loggia
}




\title{
Errata: Mapping soil water content under sparse vegetation and changeable sky conditions: comparison of two thermal inertia approaches
}

\author{
Antonino Maltese, Fulvio Capodici, Giuseppe Ciraolo, and \\ Goffredo La Loggia \\ Università degli Studi di Palermo, Dipartimento di Ingegneria Civile, Ambientale, Aerospaziale, \\ dei Materiali, Palermo 90128, Italy \\ antonino.maltese@unipa.it
}

[DOI: 10.1117/1.JRS.7.079997]

The title of this article [J. Appl. Remote Sens. 7(1), 073548 (Jun 04, 2013)] contained an error. The word "map" has been changed to "mapping," as shown above. All online versions of the article were corrected on 11 June 2013. 